\title{
Impact of Spectrum Aggregation Technology and Frequency on Cellular Networks Performance
}

\author{
Mohammed Alotaibi ${ }^{1,2}$, Marvin Sirbu ${ }^{1}$, and Jon Peha ${ }^{1}$ \\ ${ }^{1}$ Department of Engineering and Public Policy, Carnegie Mellon University, Pittsburgh, PA, USA \\ ${ }^{2}$ Communications and Information Technology Commission, Riyadh, Saudi Arabia \\ E-mails: \{alotaibi, sirbu, peha\}@cmu.edu
}

\begin{abstract}
Spectrum Aggregation (SA) technology has been introduced in cellular standards: Long Term Evolution (LTE)Advanced is expected to aggregate multiple Component Carriers (CC) to fulfill the high data rate requirement. In this paper, we use simulation to compare the performance of a cellular system using spectrum aggregation to systems that operates carriers independently, and to systems with contiguous spectrum. We vary a large number of assumptions to generate a comprehensive picture of spectrum aggregation's impact on cellular network performance. Under spectrum aggregation, users are assigned resource blocks from across all carriers. For Independent Carrier (IC) systems we model a simple carrier selection technique, which assigns a single carrier to each user. To fully understand the impact of SA, spectrum allocation is varied from contiguous to fragmented carriers across bands ranging from UHF to $2.6 \mathrm{GHz}$. This creates multiple network scenarios including intra-band and inter-band spectrum aggregation systems. Results show that LTE systems that use spectrum aggregation over fragmented blocks of spectrum can have better performance compared to independent carrier systems. Moreover, at low inter-site distances, the performance of interband SA is equivalent to that of contiguous low band spectrum, and superior to contiguous high band spectrum.
\end{abstract}

Index Terms-spectrum aggregation, carrier aggregation, LTE, spectrum allocation, independent carrier, system throughput

\section{INTRODUCTION}

$\Delta \mathrm{S}$ a cellular provider acquires more spectrum in any given Aregion, the provider may end up with a few large contiguous blocks of spectrum, or many small non-contiguous spectrum fragments, depending in part on the strategy adopted by this particular provider, and in part on the spectrum policies established by the regulator. Until recently, a provider with multiple non-contiguous spectrum blocks would operate each block as an independent resource, and each active cellular device would be served over one of these spectrum blocks at any given time. This is referred to as independent carrier operations. The fact that a cellular provider can obtain better performance over one large contiguous block of spectrum than it can when using the independent carrier approach over multiple non-contiguous blocks of equal total bandwidth has caused cellular providers to favor large blocks, and has caused regulators to go to great lengths to make large spectrum blocks available.

However, a technology called spectrum aggregation has emerged with the potential to change both the strategies by which cellular providers acquire spectrum and the policies established by regulators. Spectrum Aggregation (SA) technology, also referred to as Carrier Aggregation, combines contiguous or non-contiguous spectrum fragments to create a virtual wideband channel. It allows multiple small fragments of spectrum to be utilized to provide high transmission rate broadband services even though none of the individual fragments could support as high data rates without aggregation. Spectrum aggregation capability has now been incorporated in new mobile wireless standards, such as LTE-A and IEEE $802.16 \mathrm{~m}$, which require wideband channels to meet the high data rate requirements set by the ITU for International Mobile telecommunication - Advanced (IMT-A) standards. (Since LTE is the most promising IMT-A technology and the most adopted one across the world, the focus in this paper will be on LTE-based systems.)

The goal of this paper is to determine the level of performance that is achievable when using SA over fragmented spectrum, and compare that to the performance achievable with IC over fragmented spectrum, and the performance achievable over contiguous spectrum. Moreover, we explore the performance impact of SA in both the intraband case, wherein the spectrum blocks held by a cellular provider differ little in frequency, and the increasingly important inter-band case, where frequencies different greatly.

To the extent that SA can overcome the performance disadvantages of spectrum fragmentation, this can help regulators address a difficult challenge. Traffic on wireless mobile networks is forecasted to increase more than 10 -fold over the next five years [1]. Moreover, video traffic, which requires a high bit rate, will generate most of this growth. It is difficult to meet the performance needs of high-bit-rate video streams in narrowband spectrum fragments. The easiest way for regulators to make more spectrum available to carry all this traffic is to scour the spectrum bands for any slices that can easily be repurposed for cellular. However, this approach increases spectrum fragmentation. This may make it more difficult for carriers to obtain contiguous blocks of spectrum large enough to efficiently carry large amounts of video 
traffic. If SA is not sufficiently effective, spectrum fragmentation can result in an inefficient use of spectrum [2]. This fragmentation in spectrum available for mobile services might delay rolling out and expanding mobile broadband services like LTE [3], [4]. Alternatively, regulators could release new spectrum for cellular only in large contiguous blocks, but this approach is likely to be slower, and more costly. If SA technology becomes more cost-effective, then the former approach becomes more attractive. This paper will inform that decision.

If SA proves to be effective, this would also affect the strategy of cellular providers. For example, with effective inter-band SA, a provider is more likely to acquire a mix of high and low-frequency spectrum, rather than seeking spectrum primarily in the same low-frequency band. Bands designated for mobile services are now spread across $4 \mathrm{GHz}$ of spectrum, and each band is subdivided further into blocks for different licensees.

We start by providing background information on SA and previous work. This is followed by an overview describing our modeling approach. Next, we present a detailed view of the simulation model used. After that, we list the main inputs and the assumptions we made in the network simulation. Finally, we plot and discuss the results of the SA impact.

\section{LITERATURE REVIEW}

Spectrum aggregation technology was discussed for the first time in 2006 in a report prepared for Ofcom [2]. A demonstration of carrier aggregation started in early 2012 [8] and implementation of the technology in LTE networks started in the second half of 2013 [5] [6].

A survey and tutorial overview for radio resource management of SA in LTE-A is presented in [7] and [8]. Radio Resource Management (RRM) involves scheduler structure, Component Carrier (CC) selection and Resource Block (RB) selection. There are three scenarios for carrier aggregation: intra-band contiguous, intra-band non-contiguous and inter-band noncontiguous.

By the end of 2012, LTE networks were on air in many countries worldwide. However, LTE (Release 8) doesn't meet IMT-A requirement which is considered the true $4 \mathrm{G}$ standard. The 3GPP addressed this requirement in LTE-A with a CA feature [9]. By means of CA, users can have access to a total bandwidth of up to $100 \mathrm{MHz}$ in order to meet the IMTAdvanced requirements [10], [11]. Before this introduction, carrier aggregation was only possible for two contiguous carriers in the Dual Carrier HSPA systems [12]. CA has been introduced gradually starting from LTE Release 10 which requires multiple changes in LTE protocols for the radio, as described in [7], [13], [14] and [15].

Qualitatively, the benefits of spectrum aggregation have been discussed in different articles. First, SA can combine low-frequency bands that are used for Frequency Division Duplexing (FDD) with high-frequency bands that are used for Time Division Duplexing (TDD) to provide high-speed mobile broadband across large areas [16]. If an operator targets $5 \mathrm{Mbps}$ for High Definition (HD) video streaming, the operator can offer this service over a broader portion of the network with carrier aggregation than without. Also, SA allows the carrier to better utilize spectrum assets as a whole. It will allow peak target data rates in excess of $1 \mathrm{Gbps}$ in the downlink and $500 \mathrm{Mbps}$ in the uplink to be achieved [17]. In addition to 3GPP standards, the IEEE 802.22 standard discusses channel bonding and aggregation [18]. Spectrum aggregation and multi-user MIMO: Real-World Impact (SAMURAI) is a European project that was initiated to study this technology [19]. It runs different analyses with different scenarios to test this technology in practice and it discusses SA at link level with implementation challenges and the system aspect of SA [20], [21]. Similarly, Wireless World Initiative New Radio (WINNER+) discusses this technology in their recent reports [22].

The available frequency bands are usually non-contiguous, which may cause impact on the spectrum utilization efficiency (SUE) [23]. There are studies that investigate the spectrum fragmentation and determine the acceptable level of fragmentation for different systems [24]. One popular implementation for SA systems can be done with cognitive radios for systems with dynamic spectrum access to fragmented spectrum [25]. New interleaving schemes for downlink OFDMA of LTE-Advanced systems are proposed to capture CA-specific enhanced frequency diversity with simple implementation [26].

Furthermore, discussion in [27] includes the concept of primary cell (PCell) and secondary cell (SCell), mechanisms for activation and deactivation of CCs in cross-CC scheduling. Sometimes, they are called primary carrier and secondary carrier. Handover over the two carriers is discussed in [28]. It depends on the scheduling algorithm, but typically users are assigned RBs in the primary carrier and when more capacity is needed, RBs in the secondary carriers are aggregated.

Finally, many papers have analyzed the performance of SA through network simulations [29]. Some of the papers apply aggregation in the uplink channel [30]. In both downlink and uplink, SA can show performance improvement over operating carriers independently. [31] shows performance system improvement when SA is used versus Independent Carrier (IC). [32] analyzes Independent Carrier operation over two bands, one exclusively assigned and the other shared. Results in [33] and [34] show that the blocking rate intra-band SA system is better when cross carrier joint scheduling technique is used. While [37] discusses inter-band cases with and without spectrum aggregation in order to measure SA improvement of the performance. Overall, these papers and others analyze different scenarios of spectrum aggregation networks that use HSPA or LTE-A, inter-band and intra-band SA scenarios, and many scheduling methods. Results in general show improvement in performance due to SA. However, none of these studies provide a comprehensive comparison between systems that use SA over non-contiguous spectrum, systems that use non-contiguous carriers independently, and systems that utilize contiguous spectrum, nor have these studies explored how this comparison is affected by frequency, or the use of multiple bands at different 
frequencies. This paper will perform these comparisons.

\section{CARRIER SELECTION AND SCHEDULING}

When we compare the performance achieved with spectrum aggregation, independent carriers, and contiguous spectrum we must adopt resource allocation strategies that are appropriate for the scenarios we consider. This includes scenarios where cellular providers are using spectrum blocks at very different frequencies. In the case of independent carriers, a device must be assigned to a particular carrier when communications are established. It is the carrier selection algorithm that makes this selection. Once a connection is established, a scheduling algorithm determines which device may transmit in which resource block during the upcoming frame, where a resource block consists of 12 OFDM subcarriers transmitting for the duration of one LTE Slot $(0.5$ msec).

In a conventional LTE system operating over one carrier, the scheduler applies its algorithm directly to the single carrier available by assigning different resource blocks to different users. Introducing multiple carriers requires a careful modification to the assignment process to deal with it correctly.

\section{A. Joint Scheduling for Spectrum Aggregation}

Spectrum-aggregation should allow a cellular system to assign resource blocks from multiple carriers to the same user simultaneously. Previous researchers have considered a variety of scheduling approaches for systems that support SA. This includes Joint Queue Scheduler [31], [33] and [34], which is a one-step scheduler with single queue which should lead to the optimum performance. Alternatively, Disjoint Queue Scheduler [31], [33] and [34] is a two-step scheduler where first the primary carrier is selected, then the resource block is assigned. Its performance is lower than JQS but it is less complex. In this work, we use the joint scheduling because it has been shown to work efficiently in LTE systems [35]. With this approach, the scheduler will effectively see only one single carrier that has resource blocks equal to the sum of all resource blocks from all the carriers. Then the scheduler applies its scheduling algorithm to assign resource blocks to specific users using a generic measure of the quality of service that might be possible with that resource block, but without any explicit consideration of which carrier the resources block is associated with. In LTE, this quality measure is known as the Channel Quality Indicator (CQI).

\section{B. Carrier Selection for Independent Carriers}

In independent carrier operations, the scheduler can assign resource blocks to a given user from only a single carrier. Thus, resource allocation is a two-step process:

Step 1: select a carrier for a given user

Step 2: assign resource blocks within that carrier

To provide a fair comparison, we need a scheduling algorithm that works well in both the inter-band and intraband cases, which means the algorithm should take frequency band into consideration. More research has been done on selection algorithms for the simpler intra-band case, in which there is little difference in how well the carriers can serve any individual device, no matter where that device is located. In this case, performance is improved through some form of load balancing. An effective example is device Least Load scheduling [31], [33], [36], which selects the CC with lowest load or shortest queue in an attempt to balance load on CCs.

However, load-balancing algorithms work poorly in the inter-band case. The G-factor algorithm was introduced superficially to deal with inter-band SA when some CC can't serve users at the cell-edge. G-factor based selection [37] identifies cell edge users and allocates them the CC's with the best quality, which are generally those at the low frequencies.

To build on the best features of the algorithms above, we introduce a simple algorithm that is a hybrid of the least load and G-factor based selection algorithms. We use the Channel Quality Indicator for each user and each carrier in the selection algorithm. If the average CQI from multiple carriers for a given user is below a certain threshold, then the carrier selected will be the one with lowest frequency. Otherwise, the least load algorithm is applied. Here are the steps of the simple algorithm:

- Estimate the average CQI for each UE $j$ across all $N$ carriers as: $\mathrm{CQI}_{\mathrm{ave}}^{j}=\sum_{j=1}^{N} \frac{\mathrm{CQ}_{\bar{I}}}{N}$

- Set a threshold CQI value ( $\left.\mathrm{CQI}_{+\mathrm{H}}\right)$ to identify cell edge user, such that user $i$ is identified as cell edge user if: $C Q I_{\text {ave }}^{j}<C Q I_{\text {th }}$

- For all UEs:

○ If $U E_{j}$ is cell edge user, select the lowest

frequency carrier.

- Otherwise, select carrier with lowest number of users

\section{Scheduling Algorithm}

After we form a single queue for joint scheduling in SA or select a carrier for each user and form multiple queues in IC, we apply a scheduling algorithm to assign RBs to users. Many scheduling algorithms for SA have been proposed; Cross-CC PF [36] which collects user previous throughput information and selects CCs based on users CA capability. It increases fairness and exploits SA advantage. User grouping PF [38] divides users according to how many CCs they can be served by, then start selections. In general, there is a trade-off between performance and fairness when designing a scheduling algorithm. For example, an algorithm that simply maximizes throughput would assign most of the resource blocks to devices that are closest to a cell tower, and thus can get a higher data rate with any given resource block, while devices at the edge of the cell may starve. This disparity could be even greater in an inter-band scenario, where devices close to the cell tower dominate use of the low-frequency bands. 
For this simulation, we choose to apply the well-studied Proportional Fair (PF) scheduling algorithm [39] [40]. With this scheduling algorithm, users compete for resource blocks not based on their requested rates exclusively. Instead they compete after normalization by their respective average throughputs. The user with better channel quality will have a higher average throughput.

\section{PROBLEM FORMULATION}

Cellular system performance can be measured using different metrics. There are different measures such as fairness and latency, but the most common measure is throughput, which will be the focus of our analysis. Throughput is the actual rate that information is transferred. Cell throughput depends on multiple factors including: spectrum bandwidth, signal-to-noise ratio, cell dimension, user locations, spectral efficiency and scheduling. These factors are inter-independent and some of them are not constants, making it hard to derive throughput analytically. Instead, network simulation is used to predict throughput more precisely.

In the simulator we adopted, the network being simulated has a conventional macro-cell layout. In the center of each cell, a single Evolved Node B (eNodeB) serves UEs within the cell. In our system model, we assume an LTE-A technology based network that has been allocated a total bandwidth of $B$ $\mathrm{MHz}$ with a frequency-reuse factor of 1 . This allocated spectrum could be either a contiguous $\mathbb{E} \mathrm{MHz}$ block or composed of non-contiguous $N$ fragments, each of which has a bandwidth of $B_{i} \mathrm{MHz}$, such that $\sum_{i=1}^{N} B_{i}=B \mathrm{MHz}$. It could be allocated in any of several frequency bands designated for IMT, spanning frequency bands between $450 \mathrm{MHz}$ and 3.6 $\mathrm{GHz}$. Based on this, we analyze the following five different network scenarios:

1. a network that uses contiguous carriers within a single band of either $700 \mathrm{MHz}$ or $2600 \mathrm{MHz}$.

2. a network that uses non-contiguous carriers independently within a single band--either two or four carriers in either the $700 \mathrm{MHz}$ band or $2600 \mathrm{MHz}$ band.

3. a network that uses non-contiguous carriers independently across multiple bands (multiband)-either one or two carriers in the $700 \mathrm{MHz}$ band and one or two carriers in the $2600 \mathrm{MHz}$ band.

4. a network that aggregates non-contiguous carriers within a single band (intra-band aggregation)--either two or four carriers in either the $700 \mathrm{MHz}$ or 2600 $\mathrm{MHz}$ band.

5. a network that aggregates non-contiguous carriers across multiple bands (inter-band aggregation), one or two in the $700 \mathrm{MHz}$ band and one or two in the 2600 $\mathrm{MHz}$ band

The systems to be investigated are distinct from each other in at least one of the following ways:

- Spectrum allocated: across one or multiple bands and allocated to contiguous or non-contiguous carriers.

- Spectrum operations: component carriers can either be aggregated or operate independently
These differences will affect certain characteristics of a wireless system that will impact the system performance as a whole. This section will discuss the affected characteristics: path loss, trunking efficiency and diversity.

\section{A. Path loss}

Path loss is the reduction in transmitted signal power as the signal propagates in space. It is used in link-budget calculations to determine the effective coverage area of a transmitter. The path loss depends on frequency band, environment and distance, and it can be predicted using different propagation models. Path loss is also affected by slow fading, which is assumed not to change during the course of the simulation, and fast fading, which does.

\section{B. Trunking Efficiency}

When a set of devices all use a shared capacity, they can do so more efficiently than when that capacity is divided, and each device can only access some of the available capacity [43]. This is known as trunking efficiency. It is one reason why performance is expected to be better with contagious spectrum or SA than with IC. This can be explained with this simple example. Assume a bursty transmission system with $M$ equal bandwidth carriers over each carrier can be represented as simple $M / M / 1$ queuing system with $\lambda$ arrival rate for each carrier and $\mu$ departure rate for each carrier. The average system delay is:

$$
T=1 /(\mu-\lambda)
$$

This is the delay experienced by users in each of the $M$ carriers, when the carriers are operated independently with $M$ different queues [31]. When the carriers are aggregated, it creates a one queue system with $M \lambda$ arrival rate and $M \mu$ departure rate. This makes the average system delay:

$$
T_{S A}=1 / \mu(\mu-\lambda)
$$

This shows a clear improvement in system performance.

\section{Diversity}

The concept of multi-user diversity states that in a large system--with multiple carrier paths from cell tower to users fading independently--at any time, there is likely to be at least one carrier that is better for a particular user. With spectrum aggregation, there are more users and more channels for the scheduler to choose from, so there are more opportunities to match channels and users in a way that yields high throughput. In contrast, with independent carriers, there are multiple schedulers, each with fewer users and fewer channels.

\section{LTE SIMULATION MODEL}

We determine the achievable performance for the five network scenarios described in the previous section through a Monte Carlo network simulation. Following 3GPP guidelines [13] for analyzing an LTE system, a small-scale 19 hexagonal-cell network is adequate to test system performance. This deployment is a trade-off between low complexity and accurate interference analysis since the site being analyzed is surrounded by two rings $(6+12=18)$ of interfering sites.

Based on the simulation of traffic and user distribution, the received signal power can be estimated and, consequently, the 
LTE cell coverage and capacity can be predicted. Figure 1, below, shows a flow diagram of the analysis. We will now explain each block in detail.

\begin{tabular}{|c|c|c|c|c|}
\hline \multicolumn{2}{|r|}{ Inputs } & Model & \multicolumn{2}{|l|}{ Outputs } \\
\hline$\frac{5}{\sqrt[\pi]{\pi}}$ & $\begin{array}{l}\text { - Spectrum Allocated (CC vs. NCC \& SB } \\
\text { vs. MB) } \\
\text { - Operation (SA vs. ICC) } \\
\end{array}$ & \multirow{5}{*}{$\begin{array}{l}\text { Vienna LTE Simulator } \\
\text { - System Level } \\
\text { Simulator developed } \\
\text { by the Technical } \\
\text { University of Vienna } \\
\text { - Implemented using } \\
\text { MATLAB } \\
\text { - offer a degree of } \\
\text { flexibility for easy } \\
\text { adding of new } \\
\text { functionalities }\end{array}$} & $\begin{array}{c}\text { Average } \\
\text { Throughput }\end{array}$ & \multirow{3}{*}{$\begin{array}{l}\underset{n}{n} \\
\stackrel{0}{0} \\
3\end{array}$} \\
\hline$\underset{\text { ț }}{\tilde{C}}$ & \multirow{2}{*}{$\begin{array}{l}\text { - Technical Parameters of the LTE } \\
\text { Network }\end{array}$} & & $\begin{array}{c}\text { Peak } \\
\text { Throughput }\end{array}$ & \\
\hline రั & & & Fairness & \\
\hline \multirow{2}{*}{$\frac{\frac{\pi}{0}}{\frac{\pi}{\frac{\pi}{2}}}$} & \multirow{2}{*}{$\begin{array}{l}\text { - Scenario specific parameters } \\
\text { - Environment (urban/rural), } \\
\text { active users, inter-site distance, } \\
\text { Traffic models, scheduling, load } \\
\text { factor }\end{array}$} & & Cell-edge & \multirow{2}{*}{ 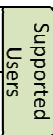 } \\
\hline & & & High bit rate & \\
\hline
\end{tabular}

Figure 1: Main blocks providing an overview of the analysis

We built our LTE simulation on top of platforms developed at the Technical University of Vienna (TU Wien) [41]. The Vienna LTE system level simulator model was implemented using MATLAB and consists of many files and functions.

We established some modifications in order to be able to run the spectrum aggregation scenarios, as summarized in Figure 2. The first column shows the basic function executed by the simulator. The second column shows the default value in the original simulator and the third column explains the changes in high-level description. In the following subsection, we explain these modifications in more details.

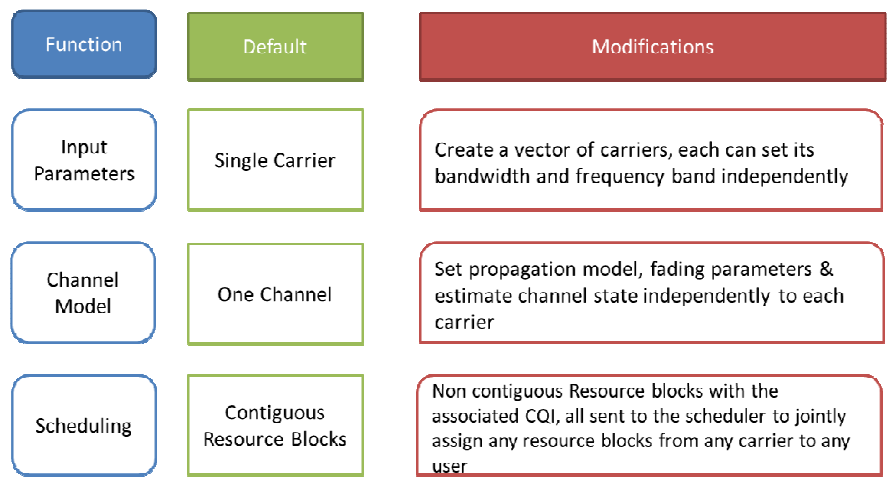

Figure 2: High-level description of modification done to the simulator

As noted earlier, spectrum-aggregation technology aggregates more than one carrier. Each carrier has its own frequency band and bandwidth. The simulator allows the user to define a single value for the two variables frequency $(f)$ and bandwidth $(B)$ both in MHz. Instead, we modified the simulator to accept multiple values for both variables, in which each pair represents values for a single carrier. This definition assumes that we can allow any device to access any resource blocks belonging to any carrier which makes the total bandwidth virtually contiguous.

Then, the standard Vienna simulator will estimate the channel state information across the cell by calculating the path loss and fading maps for that cell. Below $1500 \mathrm{MHz}$, path loss is calculated using the Okamura-Hata model; Cost231Hata is used for higher frequencies [42]. Path loss and shadow fading are calculated across the cell to generate network maps that are assumed to be valid for all carriers, which is reasonable if and only if all carriers are at roughly the same frequency. In the modified simulator, the process is repeated separately for each frequency carrier, so each carrier will have its path loss, shadow fading and small-scale fading maps. When the simulator estimates the signal to interference and noise ratio (SINR) for each UE, modifications to the simulator allow the calculation and saving of multiple SINR's for the same user for subcarriers across all different frequency carriers.
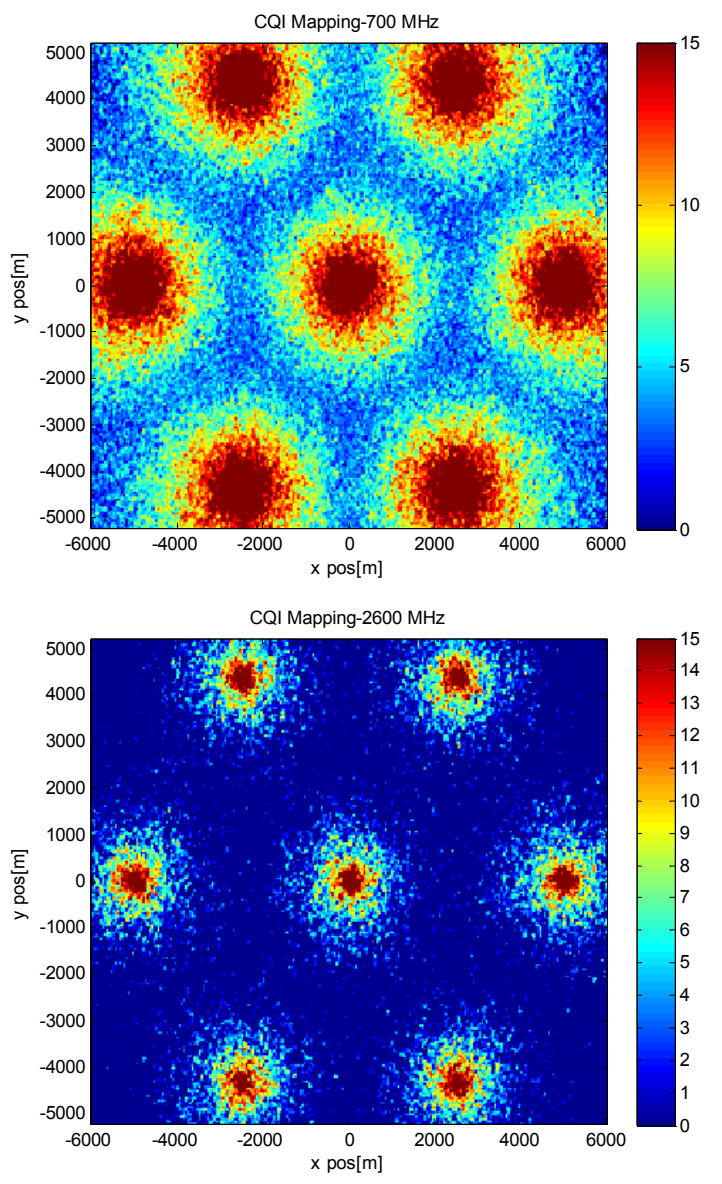

Figure 3: CQI mapping across the cell for two frequency carriers

SINR distributions for two carriers at two inter-site distances are different. For the $2600 \mathrm{MHz}$ carrier, the SINR mean across the cell drops from $9 \mathrm{~dB}$ to $-9 \mathrm{~dB}$ as we increase the inter-site distance from $500 \mathrm{~m}$ to $5 \mathrm{~km}$, while the drop is only $4 \mathrm{~dB}$ from $9 \mathrm{~dB}$ to about $5 \mathrm{~dB}$ for the $700 \mathrm{MHz}$ carrier. At a low inter-site distance both frequency carriers have almost identical distributions. When we combine subcarriers from both frequency bands, the resulting SINR distribution has cost to $0 \mathrm{~dB}$ for the $5 \mathrm{~km}$ inter-site distance, an improvement of about $8 \mathrm{~dB}$ over the system with all subcarriers in the $2600 \mathrm{MHz}$ band exclusively. Due to the difference in SINR density between the two carriers, we can see with the $2600 \mathrm{MHz}$ carrier more UE's are assigned lower CQI's as Figure 3 shows. The figure shows that cell sizes are different for each frequency band carrier. This is an example to show that a user at a distance around $2 \mathrm{~km}$ from the cell 
center might be served by one carrier but out of range of the other in a case of inter-band spectrum aggregation.

Finally, because the system we simulate has multiple carriers, we modified the initialization process to allow UEs and eNodeBs to read from multiple maps that have been generated and to store multiple CQI values, one for each set of resource blocks. Moreover, we introduced a variable that identifies which carrier each RB belongs to, for use in scheduling.

\section{INPUTS AND ASSUMPTIONS}

\section{A. Main Inputs}

Table I shows the main input assumptions concerning the allocated spectrum carriers and the way they are being operated [13]. While the total bandwidth is set to $B \mathrm{MHz}$ for each network, the differences in the number of fragments, their frequency bands and whether they are aggregated or operated independently will create the five different scenarios we will simulate.

\section{B. $\quad$ Variable Inputs}

We vary some network parameters depending on the target output in order to see the impacts.

\section{1) Traffic Models}

Depends on the output we are looking for, we alternate between finite buffer and full buffer traffic models [37].

2) Environment

The environment could be rural, suburban or dense urban. Changing this input will affect the signal propagation models and population density.

\section{3) Active users}

We vary the number of users per sector from 10 to more than 200 users based on the selected environment. We also change the arrival rate for the finite buffer traffic model.

\section{4) Inter-site distance}

We vary the inter-site distance, i.e. the distance between cell towers, from $200 \mathrm{~m}$ to $10 \mathrm{~km}$. Depending on the scheduling, users at the cell edge will be affected the most.

\section{C. $\quad$ Fixed Inputs}

Table II shows constant parameters for the network which have been inserted as inputs to the simulator. Most of the defined values were assumed based on 3GPP guidelines [13]

TABLE I

MAIN INPUTS RELATED TO FREQUENCY BANDS

\begin{tabular}{ccc}
\hline \hline Input & Value \\
\hline Total bandwidth & $20-80 \mathrm{MHz}$ \\
Block bandwidth & 5,10 and $20 \mathrm{MHz}$ \\
Number of fragments & From 1 to 5 blocks. \\
& $43 \mathrm{dBm}$ for $5 \mathrm{MHz}$ bandwidth carriers, \\
Transmit power & and $46 \mathrm{dBm}$ for 10 and $20 \mathrm{MHz}$ \\
& bandwidth carriers \\
Frequency Bands & $700 \mathrm{MHz}$ & $2600 \mathrm{MHz}$ \\
Antenna Gain & $12 \mathrm{dBi}$ & $15 \mathrm{dBi}$ \\
Path loss model & Okumura-Hata & COST $231-\mathrm{Hata}$ \\
Shadow fading SD & $8.8 \mathrm{~dB}$ & $10 \mathrm{~dB}$ \\
\hline \hline
\end{tabular}

for LTE simulation and default values that were set in the Vienna simulator.

\section{Simulation Results}

In this section, we report our results for urban environment systems.

This section presents simulation results for scenarios with contiguous spectrum (two scenarios), non-contiguous independent carriers (three scenarios) and non-contiguous spectrum aggregation (three scenarios), as described in section IV. In the case of non-contiguous carriers, we assume the system has two carriers, each with a $10 \mathrm{MHz}$ bandwidth, while the contiguous carrier is assumed to have a single $20 \mathrm{MHz}$ bandwidth. We assume full buffer traffic with 20 users per sector in an urban environment. We run the simulation for the total of eight scenarios at different inter-site distances ranging between $500 \mathrm{~m}$ and $7 \mathrm{~km}$.

In addition, we assume there is no need for an extra guardband other than the built-in LTE guardbands for both contiguous and fragmented systems. Because the LTE standards posit guardbands proportional to channel bandwidth, the intra-band SA scenarios cases have the same exact

TABLE II

SiMULATION PARAMETERS

\begin{tabular}{cc}
\hline \hline Input & Value \\
\hline Antenna Configuration & $2 \times 2$ MIMO \\
Transmission Mode & CLSM \\
TTI length & $0.001 \mathrm{~s}$ \\
Simulation Time & $1000 \mathrm{TTI}$ \\
RB Bandwidth & $180 \mathrm{kHz}$ \\
Noise figure & $9 \mathrm{~dB}$ \\
Thermal noise density & -174 \\
UE distribution & Uniform \\
Antenna azimuth offset & 30 \\
Antenna downtilt & 8 \\
Feedback channel delay & $3 \mathrm{TTI}$ \\
SINR averaging algorithm & MIESM \\
Sectors per cell & 3 \\
UE antenna gain & $0 \mathrm{~dB}$ \\
Channel model & Winner II+ \\
Channel Trace length & $5 \mathrm{~s}$ \\
Coupling loss & $20 \mathrm{~dB}$ \\
UE speed & $5 \mathrm{~km} / \mathrm{h}$ \\
Site height & $20 \mathrm{~m}$ \\
Receiver height & $1.5 \mathrm{~m}$ \\
\hline \hline
\end{tabular}

performance as the contiguous two scenarios. Therefore, we will not plot the two intra-band SA scenarios in the following figures.

\section{A. Average UE Throughput}

Figure 4 shows the average UE throughput.. The plots show that at short inter-site distances, below $1 \mathrm{~km}$, the difference in path loss between different frequency bands has an insignificant effect on throughput. This is because users are close enough to the eNodeB to be served by both frequency carriers at relatively close SINR. At these small distances, we observe a small gain for SA systems of around $7 \%$ over IC systems. This gain can be attributed to the larger multiuser 
diversity of these systems, which gives the scheduler more carriers to choose from for each UE. By continuing to compare systems that use the same frequency carriers but with different cell layouts, we note that the throughput gain increases to about $40 \%$ at $7 \mathrm{~km}$ inter-site distance. This is because the multiuser diversity becomes more significant as user density decreases within a cell as a result of the increase in inter-site distance. We also see that as the distance increases, frequency becomes a more significant factor, such that systems with only $2600 \mathrm{MHz}$ carriers will have throughput equal to $35 \%$ and $45 \%$ of the throughput of systems using either only $700 \mathrm{MHz}$ carriers or both types of carriers, respectively. In addition, inter-band systems have an extra advantage of frequency selective scheduling gain.

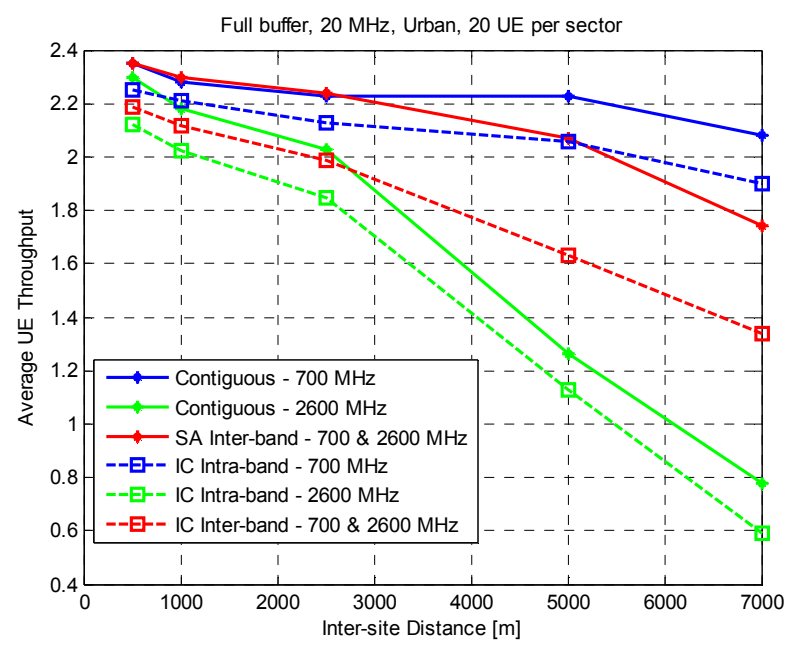

Figure 4: Average UE throughput as inter-site distance changes

When we fix the inter-site distance at $3 \mathrm{~km}$ and vary the user density per cell, we observe a number of differences in average UE throughput for each system, as Figure 5, below, shows. Moving from the $700 \mathrm{MHz}$ to the $2600 \mathrm{MHz}$ systems produces a gain of about $50 \%$ in throughput due to the advantage the lower frequency band has in wave propagation characteristics. We now look only at the two scenarios where one uses SA and the other uses IC on inter-band frequency. Again, the SA system shows approximately a $20 \%$ gain over the IC system due to multiuser diversity, which decreases as density increases. To study the diversity more closely, we change the traffic model to a finite buffer with a fixed payload size of $2 \mathrm{Mbit}$ and variable Poisson arrival rate.

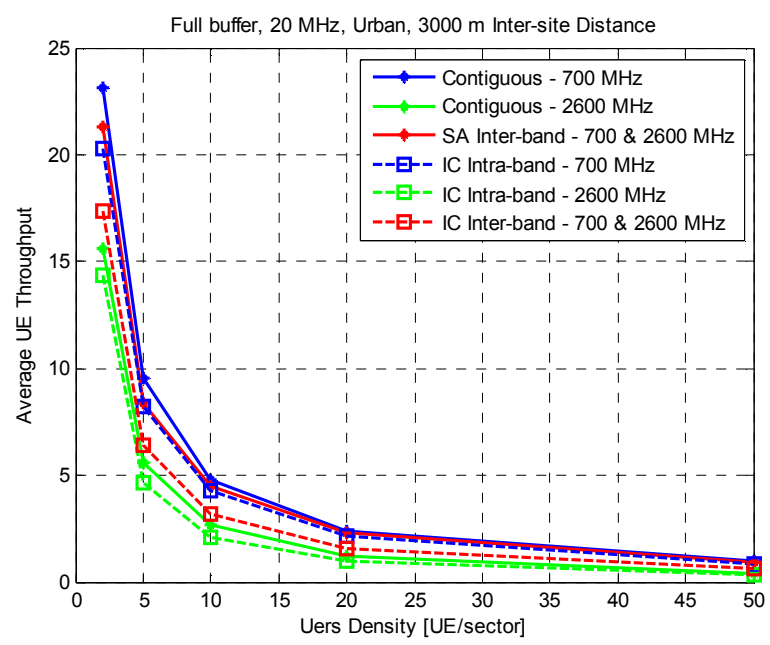

Figure 5: Average UE throughput as user density changes

Figure 6 shows that multiuser diversity combined with trunking efficiency resulting from joint scheduling provides the SA system with a gain in throughput starting from around $40 \%$ at low call arrival rates and decreasing gradually until it disappears altogether as active user density increases. This confirms previous results showing that multiuser diversity gain diminishes when user density increases.

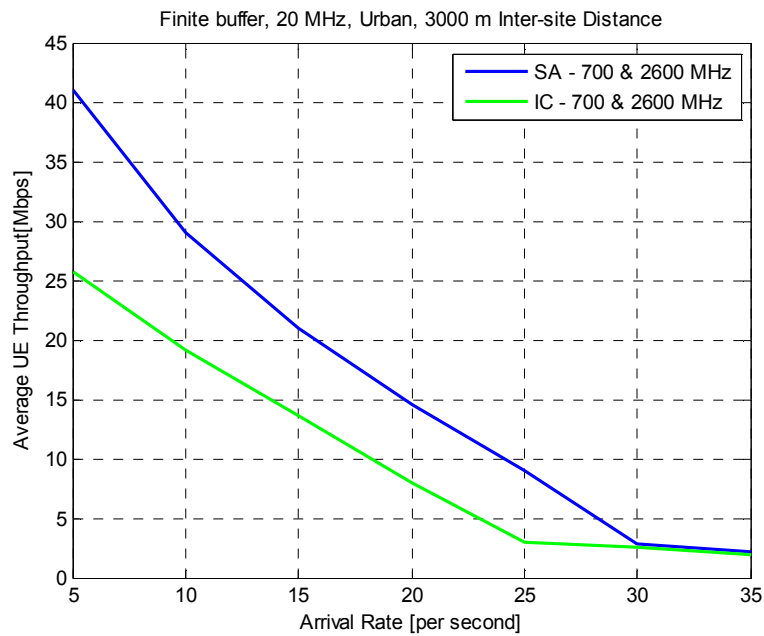

Figure 6: Average UE throughput for finite buffer traffic

\section{B. Cell Edge UE Throughput}

Looking at the edge of the cell UE throughput (Figure 7), we can see that systems with carriers only in the $2600 \mathrm{MHz}$ range will stop serving edge UE beyond approximately $3 \mathrm{~km}$ of inter-site distance, while systems having access to a $700 \mathrm{MHz}$ carrier will be able to serve edge UE beyond as much as $7 \mathrm{~km}$ of inter-site distance. As with the average UE throughput, edge UE throughput shows some gain over IC when SA is in use. This improvement is not as large as before because both systems will ultimately assign edge users to the $700 \mathrm{MHz}$ carrier only. 


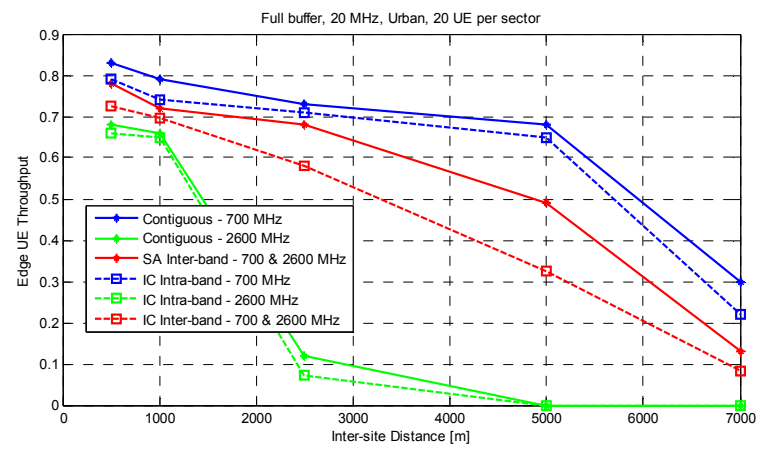

Figure 7: Throughput for UE at the cell edge

\section{Peak UE Throughput}

Looking at the peak UE throughput in Figure 8 we can see that SA systems will have at least double the peak rate that can be achieved by IC systems at cells with low density. As the IC system has access to only a more fragmented spectrum, it will realize lower peak UE throughput. As the user density increases, these differences become less significant because the peak UE throughput is reduced due to users sharing RBs. Note that frequency bands are less of an issue in the peak UE throughput simulation because this analysis focuses only on users in close proximity to the eNodeB.

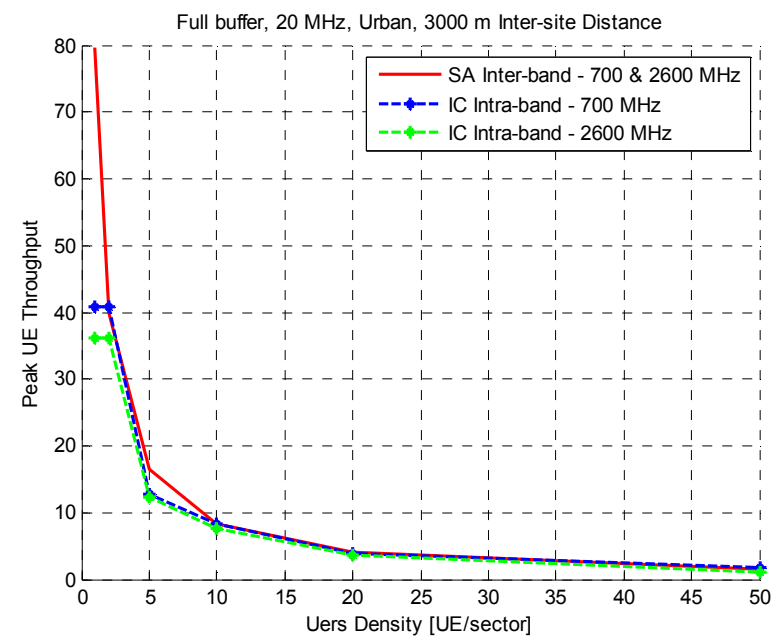

Figure 8: Peak UE throughput and impact of fragmentation

\section{Fairness}

To provide another measure that can help us understand the performance of these different network scenarios, we try to measure the fairness for each scenario. Figure 9, below, shows that the possibility of equal access is significantly affected by the frequency band(s) each system can access as inter-site distance increases. While all systems have similar fairness indicators (between 0.7 and 0.8 ) when the cell radius is small, systems that utilize only $2600 \mathrm{MHz}$ will have a low fairness rating as the cell gets larger because users at the edge of the cell cannot be served. Having access to a lower frequency band carrier is necessary to maintain good service; otherwise, the operator will be forced to build more cells with small radii.

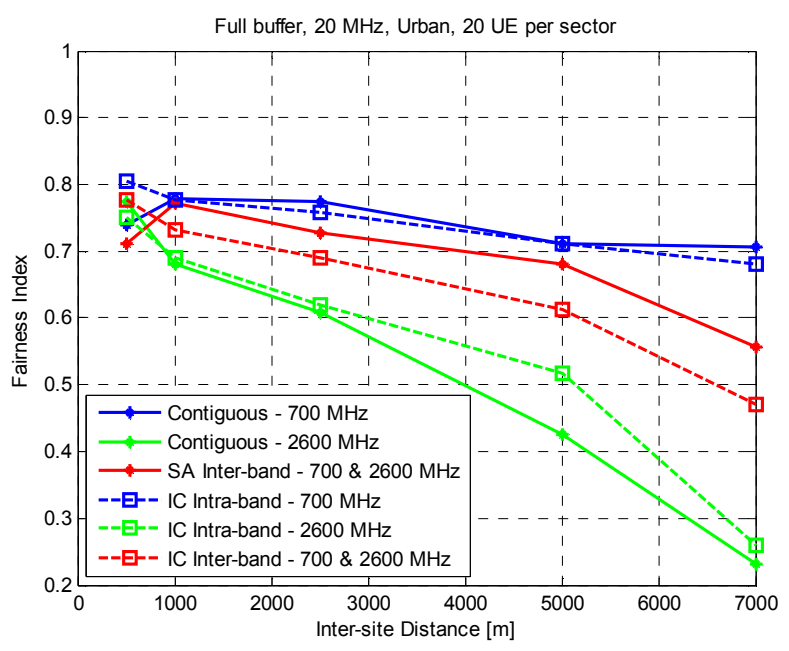

Figure 9: Measuring fairness at different cell sizes

\section{CONCLUSION}

Results from analysis in this paper show that intra-band SA and contiguous carrier systems that use the same frequency band will achieve the same performance in an LTE system as long as no additional guardband is needed to avoid interference with adjacent channels. Also, an SA system will have better performance than an IC system due to multiuser diversity and scheduling gains. These gains are more significant when the user density is low and the system isn't crowded. This applies to both full- and finite-buffer traffic models. We note that having access to a lower-frequency band carrier is necessary to maintain reasonable performance when inter-site distances are fairly large. Otherwise, cell sizes will be limited and can't be expanded.

In conclusion, this paper measured the impact of spectrum aggregation technology on LTE network performance and how this impact is affected by spectrum allocated to the operator. We show that the use of SA technology with noncontiguous carriers in multiple bands can result, at some intersite distances, in a performance level which is close to the best case scenario of using contiguous spectrum allocated in a relatively low frequency band.

\section{REFERENCES}

[1] Cisco, "Cisco Visual Networking Index: Global Mobile Data Traffic Forecast Update, 2012-2017," White Paper, 2013.

[2] Shukla, Anil; Willamson, Brian; Burns, John; Burbidge, Eddie; Taylor, Alan; Robinson, David, "A Study for the Provision of Aggregation of Frequency to Provide Wider Bandwidth Services," QinetiQ Ltd, August 2006.

[3] B. Har-Even, "LTE spectrum fragmentation delaying LTE roll out report suggests," telecoms.com, 2 August 2011. [Online]. Available: http://www.telecoms.com/31428/ltespectrum-fragmentation-delaying-lte-roll-out-report-suggests/. [Accessed 8 August 2011].

[4] FCC, "Annual Report and Analysis of Competitive Market Conditions With Respect to Mobile Wireless, Including Commercial Mobile Services," Sixteenth Report, FCC 13-34, 2013. 
[5] K. Hill, "Test \& Measurement: Carrier aggregation gains momentum," RCRWireless.com, July 2013. [Online]. Available: http:/www.rcrwireless.com/article/20130628/testequipment/test-measurement-spirent-carrier-aggregation/.

[Accessed November 2013].

[6] M. Saadi, "LTE Carrier Aggregation: Motivations and challenges," informa.com, July 2013. [Online]. Available: http://blogs.informatandm.com/14501/lte-carrier-aggregationmotivations-and-challenges/. [Accessed August 2013].

[7] H. Lee, S. Vahid and K. Moessner, "A Survey of Radio Resource Management for Spectrum Aggregation in LTE-Advanced," IEEE COMMUNICATIONS SURVEYS \& TUTORIALS, vol. ACCEPTED FOR PUBLICATION, 2013. [8] Z. Shen, A. Papasakellariou, J. Montojo, D. Gerstenberger and F. Xu, "Overview of 3GPP LTE-Advanced Carrier Aggregation for 4G Wireless Communications," IEEE Communications Magazine, pp. 122-130, February 2012.

[9] A. Roessler, S. Merkel and M. Kottkamp, "Carrier aggregation - (one) key enabler for LTE-Advanced," ROHDE \& SCHWARZ, 2012.

[10] K. I. Pedersen, F. Frederiksen, C. Rosa, H. Nguyen, L. G. U. Garcia and Y. Wang, "Carrier Aggregation for LTEAdvanced: Functionality and Performance Aspects," IEEE Communications Magazine, pp. 89-95, 2011.

[11] ETSI TR 136 912, "Feasibility study for Further Advancements for E-UTRA (LTE-Advanced)," 3GPP, 2012.

[12] 3GPP TR 25.825, "Dual-cell High Speed Downlink Packet Access (HSDPA) operation," 3GPP, 2008.

[13] ETSI TS 136 300, "LTE Overall description," 3GPP, 2013.

[14] C. Cox, An Introduction to LTE, Wiley, 2012.

[15] J. Wannstrom, "Carrier Aggregation explained," 3GPP, June 2013. [Online]. Available: http://www.3gpp.org/technologies/keywords-acronyms/101carrier-aggregation-explained. [Accessed 18 November 2013]. [16] S. Marek, "LTE-Advanced Status Report," FierceWireless, June 2014.

[17] R. Ratasuk, D. Tolli and A. Ghosh, "Carrier Aggregation in LTE-Advanced," in IEEE Vehicular Technology Conference, 2010.

[18] K. Johansson, J. Bergman, D. Gerstenberger, M. Blomgren and A. Wallén, "Multi-Carrier HSPA Evolution," in IEEE VTC, 2009.

[19] Samurai, "Spectrum Aggregation in Practice," Spectrum Aggregation and Multi-user MIMO: Real-World Impact, 2010.

[20] G. VIVIER, B. BADIC, A. CATTONI, H.-N. CHOI, J. DUPLICY, F. KALTENBERGER, I. Z. KOVÁCS, H. T. NGUYEN and S. SEZGINER, "Spectrum Aggregation: initial outcomes from SAMURAI project," in www.FutureNetworkSummit.eu, 2011.

[21] A. Cattoni, H. Nguyen, J. Duplicy, D. Tandur, B. Badic, R. Balraj, F. Kaltenberger and I. Latif, "Multi-User MIMO and Carrier Aggregation in 4G Systems: the SAMURAI Approach," in Workshop on 4G Mobile Radio Access Networks, 2012.

[22] M. Siebert, A. Mihovska, E. Mohyeldin, P. Nguyen, J.-P. Desbat, M. Mustonen and W. Mohr, "Strategies and technologies for spectrum utilisation and sharing aspects of
IMT," Wireless World Initiative New Radio - WINNER+, 2010 .

[23] H. Li, S. Dehnie, V. Chakravarthy, Z. Wu, Y. Ma and D. Walter, "Spectrum Utilization Efficiency of Cognitive Radio Systems with Limited Sampling Capability: The Impact of Spectrum Non-contiguity," in DySPAN, 2012.

[24] E. Coffman, P. Robert, F. Simatos, S. Tarumi and G. Zussman, "Channel Fragmentation in Dynamic Spectrum Access Systems - a Theoretical Study," arXiv:1002.3084, 2010 .

[25] Y. Li, S. K. Jayaweera and C. G. Christodoulou, "Wideband PHY/MAC Bandwidth Aggregation Optimization For Cognitive Radios," in 3rd International Workshop on Cognitive Incromation Processing, 2012.

[26] W. Qiu, H. Minn and C.-C. Chong, "Enhanced Frequency Diversity Exploitation in Carrier Aggregation for LTE-Advanced Systems," in IEEE Vehicular Technology Conference, 2011.

[27] K. I. Pedersen, F. Frederiksen, C. Rosa, H. Nguyen, L. G. U. Garcia and Y. Wang, "Carrier Aggregation for LTEAdvanced: Functionality and Performance Aspects," IEEE Communications Magazine, pp. 89-95, June 2011.

[28] X. Cao, D. Xiao and C. Xiu, "Handover performance of relaxing SCell measurement period in LTE-A with carrier aggregation," in Electric Information and Control Engineering, 2011.

[29] I. Shayea, M. Ismail and R. Nordin, "Capacity Evaluation of Carrier Agreggation Techniques in LTEAdvanced System," in International Conference on Computer and Communication Engineering, 3-5 July 2012.

[30] H. Wang, C. Rosa and K. Pedersen, "Performance of Uplink Carrier Aggregation in LTE-Advanced Systems," in IEEE Vehicular Technology Conference, 2010.

[31] L. Chen, W. Chen, X. Zhang and D. Yang, "Analysis and Simulation for Spectrum Aggregation in LTE-Advanced System," in IEEE Vehicular Technology Conference, Anchorage, AK, 2009.

[32] F. Meucci, O. Cabral, F. J. Velez, A. Mihovska and N. R. Prasad, "Spectrum Aggregation with Multi-Band User Allocation over Two Frequency Bands," in IEEE Mobile WiMAX Symposium, 2009.

[33] L. Zhang, K. Zheng, W. Wang and L. Huang, "Performance analysis on carrier scheduling schemes in the long-term evolution-advanced system with carrier aggregation," IET Communications, vol. 5, no. 5, pp. 612-619, April 2010.

[34] L. Zhang, Y. Wang, L. Huang, H. Wang and W. Wang, "QoS Performance Analysis on Carrier Aggregation Based LTE-A Systems," in IET International Communication Conference on Wireless Mobile and Computing, 2009.

[35] Y. Wang, K. Pedersen, T. Sorensen and P. Mogensen, "Utility Maximization in LTE-Advanced Systems with Carrier Aggregation," in IEEE Vehicular Technology Conference, 2011.

[36] Y. Wang, K. I. Pedersen, T. B. Sørensen and P. E. Mogensen, "Carrier Load Balancing and Packet Scheduling for Multi-Carrier Systems," IEEE TRANSACTIONS ON WIRELESS COMMUNICATIONS, vol. 9, no. 4, April 2010. 
[37] H. Wang, C. Rosa and K. Pedersen, "Performance Analysis of Downlink Inter-band Carrier Aggregation in LTEAdvanced," in IEEE Vehicular Technology Conference, 2011.

[38] S. Songsong, F. Chunyan and G. Caili, "A Resource Scheduling Algorithm Based on User Grouping for LTEAdvanced System with Carrier Aggregation," in International Symposium on Computer Network and Multimedia Technology, 2009.

[39] Z. Sun, C. Yin and G. Yue, "Reduced-Complexity Proportional Fair Scheduling for OFDMA Systems," International Conference on Communications, Circuits and Systems (Vol 2), 2006.
[40] C. Wengerter, J. Ohlhorst, A.G. Edler von Elbwart, "Fairness and Throughput Analysis for Generalized Proportional Fair Frequency Scheduling in OFDMA, Vehicular Technology Conference, 2005

[41] J. C. Ikuno, M. Wrulich and M. Rupp, "System level simulation of LTE networks," in IEEE Vehicular Technology Conference, Taipei, Taiwan, 2010.

[42] "APT $700 \mathrm{MHz}$ - Best Choice for nationwide coverage," ZTE Corporation, 2013.

[43] H. Salgado, M. A. Sirbu, and J. M. Peha, "A Narrow Band Approach to Efficient PCS Spectrum Sharing Through Decentralized DCA Access Policies," IEEE Personal Communications, vol. 4, no 1, Feb. 1997, pp. 24-34. 